\title{
HALPHEN CONDITIONS AND POSTULATION OF NODES
}

\author{
L. CHIANTINI ${ }^{1}$, N. CHIARLI ${ }^{2}$, S. GRECO ${ }^{3}$
}

\begin{abstract}
We give sharp lower bounds for the postulation of the nodes of a general plane projection of a smooth connected curve $C \subseteq \mathbb{P}^{r}$ and we study the relationships with the geometry of the embedding. Strict connections with Castelnuovo's theory and Halphen's theory are shown.
\end{abstract}

\section{Contents}

\begin{tabular}{|lr|}
\hline Introduction & 1 \\
\hline 1. Preliminaries and known results & 2 \\
\hline 2. Postulation of nodes and Castelnuovo's curves & 6 \\
\hline 3. Postulation of nodes and flag conditions & 8 \\
\hline 4. Minimal node functions and Halphen curves & 13 \\
\hline 5. Curves on surfaces of small degree & 20 \\
\hline 5.1 The case $s=2$ & 20 \\
\hline 5.2 The case $s=3$ & 22 \\
\hline $5.3 . \quad$ The case $s=4$ & 24 \\
References & 25
\end{tabular}

\section{INTRODUCTION}

This paper deals with smooth projective non-degenerate curves $C \subset \mathbb{P}^{r}$, of degree $d$ and genus $g$, and their general projections $C \rightarrow C_{0} \subset \mathbb{P}^{2}$. If $N$ is the set of nodes of $C_{0}$, it is classically known that the postulation of $N$ determines many intrinsic and extrinsic geometric properties of $C$, such as, for example, the existence of special linear series, the classical relation among $d, g$ and the degree $\delta$ of $N$ and the equality $e=d-3-\alpha$, where $e$ is the speciality of $C$ and $\alpha$ is the least degree of a curve containing $N$ (see Section 1 ).

Much subtler results show how the existence of special linear series $g_{k}^{n}$ depends on the existence of surfaces of low degree $s$ containing $C$, via complicate but sometimes sharp numerical relations between $r, n, k, s, d$ and $g$, that one can find in the literature (see [1], 2], 5], 8, 9], or 4], in which our principal procedure is settled).

The main goal of this paper is to prove some sharp lower bounds for $\partial h_{N}$, the first difference of the Hilbert function of $N$, and their relationships with the geometry of the embedding $C \hookrightarrow \mathbb{P}^{r}$. Since $\alpha$ is also the maximum of $\partial h_{N}$, this approach brings into play,

Date: July 2, 2018.

1991 Mathematics Subject Classification. 14H50.

Supported by MIUR and GNSAGA-INDAM, Italy. 
in a natural way, the speciality $e$. Indeed our bounds will be given in terms of $d, r$ and $\alpha$ (rather than $e$ ) and, when $r=3$, we consider also an integer $s$ such that $C$ is contained in no surface of degree $s-1$. These bounds are obviously related to upper bounds for $g$ in terms of $d, r, \alpha$ (hence $e$ ) and $s$. So our theory is strictly related to Castelnuovo's theory and to Halphen's theory of maximal genus, on which we hope to bring some new point of view.

The paper is organized as follows. After the preliminary Section 1, in the second section, we deal with non-degenerate curves in $\mathbb{P}^{r}$, for arbitrary $r \geq 3$ and we give a sharp lower bound for $\partial h_{N}$, depending only on degree and speciality, using the main result in [7]. The result is a Castelnuovo-like theory on the relationship between postulation of nodes and genus of curves.

In the rest of the paper we work in $\mathbb{P}^{3}$ and we bring $s$ into play. In Section 3 we give some general results which show how $s$ affects $\partial h_{N}$, the most used in the sequel being Theorem 3.5. For this we use two main tools: first we translate the Hilbert function of $N$ into the dimensions of some linear series on $C$, using the classical approach and the results contained in [7]; next we bound the dimensions of the linear series, using the procedure introduced in [4] and the results of [6].

In Section 4 we prove the key Theorem 4.2, which produces our main lower bound for $\partial h_{N}$, depending on $d, s$ and $\alpha$. As immediate but useful Corollaries we get an upper bound for $g$ (in terms of $d, s$ and $\alpha$ ) and a lower bound for $\alpha$ (in terms of $d$ and $s$ ).

Then we study the sharpness of our bounds, with the extra assumption $d>s(s-1)$. In this case our lower bound for $\alpha$ can be made explicit: thus we get a purely elementary arithmetical proof of the celebrated Halphen-Gruson-Peskine bound for $e$ (Proposition 4.8) and we can show that the curves with $\alpha$ minimal and minimal node function are exactly the Halphen curves. Indeed our approach allows an elementary proof of some known characterizations of such curves (Theorem 4.10).

The lower bounds for $\partial h_{N}$ is obtained so far when the value of $\alpha$ is minimal and the curves attaining the bound turn out to be aCM.

Our next step, developed in Section 5, is to consider minimal nodal functions with $\alpha$ non-minimal. Here the geometry becomes much more difficult, and we are able to give some results only for $s \leq 4$. The case $s=2$ is easy and we can give a complete picture. For $s=3$ and $s=4$ we show that our bounds are sharp for every $d>s(s-1)$, by producing explicitly curves on the smooth cubic surface or in the so-called Mori surfaces (see [15]). Moreover, using Theorem 3.5, we show that, under some mild numerical restriction, every curve attaining the bound must lie on a surface of degree $s$. Surprisigly the curves we produce, except for some sporadic cases, are not aCM: this makes us believe that the study of curves with minimal nodal postulation but non-minimal $\alpha$, and of their cohomology, is a challanging problem, even for low values of $s$.

\section{Preliminaries AND KNOWN RESUlts}

In this section we fix some notation and we collect some known results.

We work over an algebraically closed field of characteristic zero.

Let $C \subset \mathbb{P}^{r}, r \geq 3$, be a smooth connected non-degenerate curve of degree $d$ and genus

$g$. 
We put $n:=h^{0}\left(\mathcal{O}_{C}(1)\right)-1$, whence $C$ is a projection of a linearly normal curve of degree $d$ in $\mathbb{P}^{n}$ (i.e. of a curve in $\mathbb{P}^{n}$ which is not a projection of a curve of the same degree spanning a higher dimensional projective space). In particular $C$ is linearly normal if and only if $n=r$.

We denote by $e$ the index of speciality of $C$, namely

$$
e:=\max \left\{j \mid h^{1}\left(\mathcal{O}_{C}(j)\right)>0\right\}=\max \left\{j \mid h^{2}\left(\mathcal{I}_{C}(j)\right)>0\right\} .
$$

Recall that $C$ is said to be special if and only if $e>0$.

Let $C \rightarrow C_{0} \subset \mathbb{P}^{2}$ be a general plane projection, so that $C_{0}$ has only nodes as singularities.

Let $N \subset \mathbb{P}^{2}$ be the reduced subscheme of the nodes of $C_{0}$ and denote by $\Delta$ the pull-back of $N$ to $C$, viewed as a divisor on $C$.

If $\delta$ is the length of $N$, we have the classical relation:

$$
\delta=\frac{(d-1)(d-2)}{2}-g .
$$

Let $h_{N}$ be the Hilbert function of $N$ and let $\partial h_{N}$ be its first difference.

Finally let

$$
\alpha:=\min \left\{j \mid h^{0}\left(\mathcal{I}_{N}(j)\right) \neq 0\right\}
$$

The classical theory of adjoint curves relates the linear systems of curves passing through $N$ to the geometry of $C$. The starting points of this theory can be summarized in the following remark (for details see e.g. [7]):

Remark 1.1. Let $D$ be a hyperplane divisor of $C$. Then we have:

(i) the canonical map $H^{0}\left(\mathcal{I}_{N}(j)\right) \rightarrow H^{0}\left(\mathcal{O}_{C}(j D-\Delta)\right)$ is surjective for every $j \in \mathbb{Z}$ and also injective for $j \leq d-1$;

(ii) the canonical sheaf $\omega_{C}$ of $C$ is $\mathcal{O}_{C}((d-3) D-\Delta)$;

(iii) from (i) and (ii) it follows, by Riemann-Roch and standard calculations:

$$
\partial h_{N}(j)=j+1-d-h^{0}\left(\mathcal{O}_{C}(d-j-3)\right)+h^{0}\left(\mathcal{O}_{C}(d-j-2)\right) \quad \text { for } j \leq d-1 ;
$$

(iv) by (ii) the the map of (i) can be viewed as $H^{0}\left(\mathcal{I}_{N}(j)\right) \rightarrow H^{0}\left(\omega_{C}(d-3-j)\right)$, hence from (iii) it follows:

$$
\partial h_{N}(j)=j+1-h^{0}\left(\omega_{C}(j-d+3)\right)+h^{0}\left(\omega_{C}(j-d+2)\right) \quad \text { for } j \leq d-1 ;
$$

(v) from the above we get $\alpha=d-3-e$. It follows $\alpha \leq d-2$, with equality if and only if $g=0$. Moreover $\alpha \leq d-4$ if and only if $C$ is special.

By general properties of the Hilbert function of a zero-dimensional subscheme of $\mathbb{P}^{2}$ and by Remark 1.1 we have the following result:

Proposition 1.2. The difference function $\partial h_{N}$ satisfies:

(a) $\partial h_{N}(j)=0$ for $j<0$ and for $j>d-3$;

(b) $\partial h_{N}(j)=j+1$ for $0 \leq j \leq \alpha-1$;

(c) $\partial h_{N}$ is non-increasing for $j \geq \alpha-1$;

(d) $\partial h_{N}(d-3)=n-2 \geq r-2$. 
Proposition 1.2 above gives an accurate description of the function $\partial h_{N}$, except for the interval $[\alpha, d-4]$.

The following result ( 7 , Theorem 5.1), which holds in arbitrary characteristic, provides a lower bound for the descent of $\partial h_{N}(j)$ at any step between $\alpha$ and $d-3$. More refined bounds for $r=3$ will be given in the next sections.

Theorem 1.3. (arbitrary characteristic) For $\alpha \leq j \leq d-3$ one has:

$$
\partial h_{N}(j+1) \leq \partial h_{N}(j)-n+2 \leq \partial h_{N}(j)-r+2 .
$$

In particular $\partial h_{N}$ is strictly decreasing in the interval $[\alpha, d-2]$.

It is easy to derive some (well known) speciality bounds from the previous result.

Corollary 1.4. The following inequalities hold:

(a) $\alpha \geq(d-2-\alpha)(n-2) \geq(d-2-\alpha)(r-2)$;

(b) $\alpha \geq \frac{(d-2)(n-2)}{n-1} \geq \frac{(d-2)(r-2)}{r-1}$;

(c) $e \leq \frac{d-n-1}{n-1} \leq \frac{d-r-1}{r-1}$.

Proof. $\alpha$ is the maximum for $\partial h_{N}$. On the other hand by Theorem $1.3 \partial h_{N}$ decreases of at least $n-2$ at any step from $j=\alpha$ to $j=d-3$; furthermore $\partial h_{N}(d-3)=n-2$ by Proposition $1.2(\mathrm{~d})$ and (a) follows.

Clearly (b) is equivalent to (a). Finally (c) follows from (b) and Remark 1.1(iii).

In $\mathbb{P}^{3}$ we get back the well known speciality bound $e \leq \frac{(d-4)}{2}$, attained for instance by complete intersections on quadrics. A complete classification of the curves in $\mathbb{P}^{3}$ achieving this speciality bound will be given in Proposition [5.3 (see also Remark [5.4).

Remark 1.5. Concerning the relations between the values $\partial h_{N}(\alpha-1)=\alpha$ and $\partial h_{N}(\alpha)$ we can say the following:

(a) by Theorem 1.3 we have $0 \leq \alpha-\partial h_{N}(\alpha) \leq \alpha-(d-2-\alpha)(n-2)$;

(b) $\partial h_{N}(\alpha)=\alpha$ if $C$ is subcanonical, but not conversely. Indeed $C$ is subcanonical if and only if $\alpha d=2 \delta$. Moreover in this case there is a double structure on $N$ which is a complete intersection of type $(\alpha, \delta)$ (see [7]);

(c) if $\alpha$ is minimal with respect to $d$ and $r$, namely $\alpha=\left\lceil\frac{(d-2)(r-2)}{r-1}\right\rceil$ (see Corollary 1.4(b)), then $\alpha-\partial h_{N}(\alpha) \leq n-2$, with equality if and only if $\alpha=\frac{(d-2)(r-2)}{r-1}$. This is an easy consequence of Corollary 1.4 (details to the reader).

We end this section by showing that when $C$ is arithmetically Cohen-Macaulay (aCM) $\partial h_{N}$ can be computed from the Hilbert function of a general hyperplane section of $C$ (and conversely).

We recall first an easy fact.

Remark 1.6. Let $\Gamma$ be a general hyperplane section of $C$. Then it is easy to see that

$$
h^{0}\left(\mathcal{O}_{C}(j)\right)-h^{0}\left(\mathcal{O}_{C}(j-1)\right) \geq h_{\Gamma}(j) \text { for every } j \in \mathbb{Z}
$$

with equality whenever $h^{1}\left(\mathcal{I}_{C}(j)\right)=0$ : moreover equality holds for all $j \in \mathbb{Z}$ if and only if $C$ is aCM. 
Hence by Remark 1.1(iii) we have

$$
\partial h_{N}(j) \geq j+1-d+h_{\Gamma}(d-2-j) \text { for every } j \leq d-1
$$

with equality whenever $h^{1}\left(\mathcal{I}_{C}(j)\right)=0$; moreover equality holds for every $j \leq d-1$ if and only if $C$ is aCM.

Example 1.7. Assume that $C$ is aCM and let $\Gamma$ be a general hyperplane section of $C$. Then by Remark [1.6 we have

$$
\partial h_{N}(j)=j+1-d+h_{\Gamma}(d-2-j) \text { for every } j \leq d-1 .
$$

It is also easy to see that the index of speciality of $C$ is

$$
e=\max \left\{j \in \mathbb{Z} \mid h^{1}\left(\mathcal{I}_{\Gamma}(j)\right) \neq 0\right\}-1
$$

whence

$$
\alpha=d-2-\max \left\{j \in \mathbb{Z} \mid h^{1}\left(\mathcal{I}_{\Gamma}(j)\right) \neq 0\right\} .
$$

Then, since $\partial h_{N}(j)=j+1$ for $0 \leq j \leq \alpha-1$ it is immediate to write down $\partial h_{N}(j)$ for every $j \in \mathbb{Z}$, in terms of $d$ and $h_{\Gamma}$.

For example if $r=3$ and $C$ is a complete intersection of type $(s, s+u)$, with $u \geq 0$, we have

$$
\partial h_{\Gamma}(j)= \begin{cases}0 & \text { if } j<0 \\ j+1 & \text { if } 0 \leq j \leq s-1 \\ s & \text { if } s \leq j \leq s+u-1 \\ 2 s+u-1-j & \text { if } s+u \leq j \leq 2 s+u-2 \\ 0 & \text { if } j \geq 2 s+u-1\end{cases}
$$

It follows (as expected):

$$
e=2 s+u-4
$$

whence

$$
\alpha=d+1-(2 s+u)
$$

and, with some calculations,

$$
\partial h_{N}(j)= \begin{cases}0 & \text { if } j<0 \\
j+1 & \text { if } 0 \leq j<\alpha \\
\alpha-\left(\begin{array}{c}
j-\alpha+1 \\
2
\end{array}\right) & \text { if } \alpha \leq j \leq d-s-u-2=\alpha+s-3 \\
\left(\begin{array}{c}
s \\
2
\end{array}\right)+(d-s-1-j)(s-1) & \text { if } d-s-u-1 \leq j \leq d-s-2 \\
\left(\begin{array}{c}
d-1-j \\
2
\end{array}\right) & \text { if } d-s-1 \leq j \leq d-3 \\
0 & \text { if } j>d-3\end{cases}
$$

Remark 1.8. The Hilbert function of $N$ is strictly related with the spectrum $\ell_{C}$ of $C$, defined as (see [16]):

$$
\ell_{C}(j):=\partial^{2} h^{0}\left(\mathcal{O}_{C}(j)\right) \text { for all } j \in \mathbb{Z}
$$

Indeed from Remark 1.1(iii) it follows easily that

$$
\partial^{2} h_{N}(t)=1-\ell_{C}(d-1-t) \quad \text { for } \quad \alpha \leq t \leq d-1
$$


For example if $C$ is aCM and $\Gamma$ is a general hyperplane section of $C$ the spectrum of $C$ is

as follows easily by Example 1.7.

$$
\ell_{C}(j)=\partial h_{\Gamma}(j) \text { for } j \in \mathbb{Z}
$$

\section{Postulation of nodes and Castelnuovo's Curves}

In this section we use Theorem 1.3 to produce a lower bound for $\partial h_{N}$ in terms of $d$ and $r$ and we show that this bound is sharp for curves of maximal genus, that is Castelnuovo's curves. Moreover we give some lower and upper bounds for the length $\delta$ of $N$ depending on $d, r$ and $\alpha$, hence lower and upper bounds for $g$ depending on $d, r$ and $e$.

Proposition 2.1. Set $\beta:=\left\lceil\frac{(d-2)(r-2)}{r-1}\right\rceil$ and $q:=d-2-\beta$ and let $\phi_{\beta}$ be the function defined by:

$$
\phi_{\beta}(j)= \begin{cases}0 & \text { if } j<0 \\ j+1 & \text { if } 0 \leq j \leq \beta-1 \\ (d-2-j)(r-2) & \text { if } \beta \leq j \leq d-3 \\ 0 & \text { if } j \geq d-2\end{cases}
$$

Then we have:

(a) $\partial h_{N}(j) \geq \phi_{\beta}(j)$ for every $j \in \mathbb{Z}$;

(b) $\delta \geq \frac{1}{2}\left(\beta(\beta+1)+(r-2)\left(q^{2}+q\right)\right)$;

Moreover the following conditions are equivalent:

(i) $\partial h_{N}(j)=\phi_{\beta}(j)$ for every $j \in \mathbb{Z}$;

(ii) $\delta=\frac{1}{2}\left(\beta(\beta+1)+(r-2)\left(q^{2}+q\right)\right)$;

(iii) $C$ is a Castelnuovo curve (that is a curve of maximal genus among the non degenerate curves of degree $d$ in $\left.\mathbb{P}^{r}\right)$.

Proof. By Corollary 1.4 we have $\beta \leq \alpha$ and (a) follows by Theorem 1.3. Moreover (a) implies $\delta=\sum_{j=0}^{d-3} \partial h_{N}(j) \geq \sum_{j=0}^{d-3} \phi_{\beta}(j)=\frac{1}{2}\left(\beta(\beta+1)+(r-2)\left(q^{2}+q\right)\right)$, whence (b).

The equivalence of (i) and (ii) follows immediately from (a) and (b).

To conclude the proof we recall that Castelnuovo's curves are characterized by the equality

$$
g=\left(\begin{array}{c}
m \\
2
\end{array}\right)(r-1)+m \epsilon
$$

where

$$
d-1=(r-1) m+\epsilon, \quad 0 \leq \epsilon \leq r-2 .
$$

(see e.g. 12 or 3] for details).

Now equality in (b) is equivalent to

$$
g=\frac{(d-1)(d-2)}{2}-\frac{1}{2}\left(\beta(\beta+1)+(r-2)\left(q^{2}+q\right)\right) .
$$

It is easy to see that $q=d-2-\beta=\left[\frac{d-2}{r-1}\right]$, that is

$$
d-2=(r-1) q+\eta, \quad 0 \leq \eta \leq r-2 .
$$


This implies, by a straightforward calculation, that (3) is equivalent to

$$
g=\left(\begin{array}{l}
q \\
2
\end{array}\right)(r-1)+q(\eta+1) .
$$

Now if $\eta \leq r-3$ we have $(m, \epsilon)=(q, \eta+1)$ and if $\eta=r-2$ we have $(m, \epsilon)=(q+1,0)$. It follows that in both cases (44) is equivalent to (2).

Remark 2.2. Proposition 2.1] shows that the lower bound for $\partial h_{N}$ provided by Theorem 1.3 and the lower bound for $\alpha$ given by Corollary 1.4 are sharp, since they are attained by Castelnuovo's curves. However we will see that these bounds can be achieved also by other curves in $\mathbb{P}^{3}$, where the situation is quite clear (see Section 5 ). In higher-dimensional spaces several things are still unclear. For example even considering complete intersections of quadrics we have the following situation.

Let $C$ be a complete intersection of 3 quadrics in $\mathbb{P}^{4}$. Then $d=8$ and $g=5$, so that $C$ is a Castelnuovo curve. Indeed a direct calculation easily shows that

$$
\partial h_{N}: \ldots, 0,1,2,3,4,4,2,0, \ldots
$$

coherently with Proposition 2.1.

On the other hand, the reader can easily realize (e.g. by using Example 1.7) that complete intersections of quadrics in higher dimensional projective spaces, which are not Castenuovo's curves, do not have minimal descent of $r-2$ at any step after $\alpha+1$.

Also in $\mathbb{P}^{4}$ more general complete intersections do not have the minimal descent, as the following example shows.

Example 2.3. Let $C$ be a complete intersection of type $(2,2,3)$ in $\mathbb{P}^{4}$. Then $d=12$ and $g=13$, whence $\delta=55-13=42$.

Since $\omega_{C}=\mathcal{O}_{C}(2)$, by Remark 1.1] we have $\alpha=d-3-2=7$. Then $\partial h_{N}(j)=j+1$ for $j<7$ while $\partial h_{N}(j)=0$ for $j>d-3=9$; furthermore the sum of $\partial h_{N}$ is 42 .

It remains to compute $\partial h_{N}(j)$ for $j=7,8,9$. Theorem 1.3 says that $\partial h_{N}(8) \leq \partial h_{N}(7)-$ $2, \partial h_{N}(9) \leq \partial h_{N}(8)-2$, while $\partial h_{N}(9)=2$ by proposition 1.2. Summing up, we see that the unique possibility for $\partial h_{N}$ is

$$
\partial h_{N}: \ldots, 0,1,2,3,4,5,6,7,7,5,2,0, \ldots
$$

Observe that there is an intermediate step in which the descent is by 3 , i.e. more than the minimum allowed $r-2=2$, whence, in particular, $C$ is not a Castelnuovo curve. However $\alpha$ reaches the minimal value established by Corollary 5.3 .

Proposition 2.4. Assume $g>0$, set

$$
\begin{aligned}
& p:=d-2-\alpha, \\
& \mu:=\alpha-p(r-2),
\end{aligned}
$$

and write $\alpha$ as

$$
\alpha=(r-2) m+\nu, \quad 0 \leq \nu \leq r-3 .
$$

Then $p>0, \mu \geq 0$ and $\delta$ satisfies the following inequalities: 
(a) $\frac{1}{2}\left(\alpha(\alpha+1)+(r-2)\left(p^{2}+p\right)\right) \leq \delta \leq \frac{1}{2}\left(\alpha(\alpha+1)+(r-2)\left(p^{2}+p\right)\right)+\mu(p-1)$;

(b) $\delta \leq \frac{1}{2}\left(\alpha(\alpha+1)+(r-2)\left(m^{2}+m\right)\right)+\nu(m-1)$.

Proof. We have $p>0$ by Remark 1.1(v) and $\mu \geq 0$ by Corollary 1.4. Consider now the numerical functions $\phi$ and $\Phi$ defined by:

$$
\begin{gathered}
\phi(j)= \begin{cases}0 & \text { if } j<0 \\
j+1 & \text { if } 0 \leq j \leq \alpha-1 \\
(d-2-j)(r-2) & \text { if } \alpha \leq j \leq d-3 \\
0 & \text { if } j \geq d-2\end{cases} \\
\Phi(j)= \begin{cases}\phi(j) & \text { if } j \leq \alpha-1 \\
\phi(j)+\mu & \text { if } \alpha \leq j \leq d-4 \\
\phi(j) & \text { if } j \geq d-3\end{cases}
\end{gathered}
$$

By Theorem 1.3 and Proposition 1.2 we have that $\phi(j) \leq \partial h_{N}(j) \leq \Phi(j)$ for every $j$. Moreover $\delta=\sum_{j=0}^{d-3} \partial h_{N}(j)$, whence $\sum_{j=0}^{d-3} \phi(j) \leq \delta \leq \sum_{j=0}^{d-3} \Phi(j)$. Then (a) follows by a straightforward calculation.

To show (b) it is sufficient to show that $\frac{1}{2}(r-2)\left(p^{2}+p\right)+\mu(p-1) \leq \frac{1}{2}(r-2)\left(m^{2}+\right.$ $m)+\nu(m-1)$. Since $\mu \geq 0$ it is easy to see that $m \geq p$ and $\mu=(r-2)(m-p)+\nu$. The conclusion follows by a direct computation.

Remark 2.5. The bounds of Proposition 2.4 are equivalent to bounds for the genus in terms of $d, r$ and $e$. The easy transformation is left to the reader.

\section{Postulation of nodes and Flag Conditions}

The sharpness of the previous results is rather flabby because as soon as one takes curves which do not lie on surfaces of minimal degree, then stronger restrictions seem to apply (consider for example complete intersections of surfaces of degree at least 3 in $\mathbb{P}^{3}$, see 4.7).

So one is led to ask whether more information about the flags of subvarieties of $\mathbb{P}^{r}$ containing $C$ could give more restrictive constraints to the behavior of $\partial h_{N}$.

To obtain sharper information on the shape of $\partial h_{N}$ one may start with the formula

$$
\partial h_{N}(j)=j+1-h^{0}\left(\omega_{C}(j-d+3)\right)+h^{0}\left(\omega_{C}(j-d+2)\right)
$$

(see Remark 1.1(iv)). This formula can be read as

$$
\partial h_{N}(j)=j+1-h^{0}\left(\mathcal{O}_{C}(L+j D)\right)+h^{0}\left(\mathcal{O}_{C}(L+(j+1) D)\right)
$$

where $D$ is a hyperplane divisor and $L:=K-(d-3) D$ is a divisor corresponding to the invertible sheaf $\omega_{C}(-d+3)$. Then one is led to study the growth of the linear series $|L+j D|$ with respect to $j$, that is the growth Hilbert function $h_{L}$ of $L$, defined as:

$$
h_{L}(j):=h^{0}\left(\mathcal{O}_{C}(L+j D)\right) \text { for every } j \in \mathbb{Z},
$$

which was introduced in [2] and studied intensively by many authors. 
With this notation (5) can be rewritten as

$$
\partial h_{N}(j)=j+1-\partial h_{L}(j+1), \quad \mathcal{O}_{C}(L)=\omega_{C}(-d+3)
$$

and any result on the step-by step growth of $h_{L}$ can be translated into results on the descent of $\partial h_{N}$ from $\alpha$ to 0 , and conversely.

Equivalently, one may start from equation

$$
\partial h_{N}(j)=j+1-d-h^{0}\left(\mathcal{O}_{C}(d-j-3)\right)+h^{0}\left(\mathcal{O}_{C}(d-j-2)\right)
$$

in Remark 1.1(iii), which can be rewritten as

$$
\partial h_{N}(j)=j+1-d+\partial h_{L}(-j+1), \quad L:=(d-3) D
$$

Here information on the growth of $h_{L}$ are translated, reversing the orientation, into information on the decrease of $\partial h_{N}$, starting from $d-3$ and going backward to $\alpha$.

We will take both points of view in order to relate geometric properties of the embedding $C \subset \mathbb{P}^{r}$ to arithmetic properties of the functions $h_{L}$ above.

Remark 3.1. In order to show how this method works we give an alternative short proof (in characteristic zero) of Theorem 1.3 .

Let $M$ be a divisor corresponding to $\omega_{C}(j-d+3)$ and let $D$ be a hyperplane divisor of $C$. Then by assumption one has $h^{0}\left(\mathcal{O}_{C}(M-D)\right)>0$ for all $j \geq \alpha+1$. Let $\Gamma$ be a general hyperplane section of $C$ and look at the exact sequences:

$$
\begin{gathered}
0 \rightarrow H^{0}\left(\mathcal{O}_{C}(M-D)\right) \rightarrow H^{0}\left(\mathcal{O}_{C}(M)\right) \rightarrow H^{0}\left(\mathcal{O}_{\Gamma}\right) \\
0 \rightarrow H^{0}\left(\mathcal{O}_{C}(M)\right) \rightarrow H^{0}\left(\mathcal{O}_{C}(M+D)\right) \rightarrow H^{0}\left(\mathcal{O}_{\Gamma}\right)
\end{gathered}
$$

Let $v=h^{0}\left(\mathcal{O}_{C}(M)\right)-h^{0}\left(\mathcal{O}_{C}(M-D)\right)$ and $v^{\prime}=h^{0}\left(\mathcal{O}_{C}(M+D)\right)-h^{0}\left(\mathcal{O}_{C}(M)\right)$ be the dimensions of the images in $H^{0}\left(\mathcal{O}_{\Gamma}\right)$ of the two left hand side maps above. Then $\Gamma$ is in uniform position with respect to the linear series $|M|$ and $|D|$, so one may apply Castelnuovo's lemma of $\S 1$ in [5]. It turns outthat $v^{\prime} \geq v+r-1$. A direct computation shows that the same inequality holds also when $j=\alpha$.

Now by equality (5) above, we obtain:

$$
\begin{aligned}
\partial h_{N}(j+1)-\partial h_{N}(j)= & h^{0}\left(\omega_{C}(j-d+4)\right)-2 h^{0}\left(\omega_{C}(j-d+3)\right)+ \\
& +h^{0}\left(\omega_{C}(j-d+2)\right)-1 \\
\geq & v^{\prime}-v-1
\end{aligned}
$$

and the claim follows.

Now we go back to our main problem, namely to take into account some flag conditions in order to get sharper information on $\partial h_{N}$. As far as we know, such a theory is available only for curves in $\mathbb{P}^{3}$, where the knowledge of a number $s$ such that $C$ is contained in no surfaces of degree smaller than $s$ influences the growth of the dimensions of linear series on the curve, under the addition of multiples of $D$. Indeed let us recall a corollary of the main technical result of [6], appearing in [4] (see also [16] for generalizations to non-integral curves): 
Theorem 3.2. Let $C \subseteq \mathbb{P}^{3}$ be a smooth connected curve and let $L$ be a divisor on $C$. Let $t$ and $j$ be integers such that:

$$
\left(\begin{array}{c}
t-1 \\
2
\end{array}\right)<\partial h_{L}(j)<d-\left(\begin{array}{l}
t \\
2
\end{array}\right)
$$

Then:

$$
\partial h_{L}(j+1) \geq \partial h_{L}(j)+t
$$

Proof. See [4, Theorem 2.3.

Notice that the assumption of the above theorem implies $\left(\begin{array}{c}t-1 \\ 2\end{array}\right)<d-\left(\begin{array}{l}t \\ 2\end{array}\right)$, hence also $d>(t-1)^{2}+1$, whence by Laudal's Lemma also the general hyperplane section of $C$ is not contained in any curve of degree $t-1$.

We point out the consequences of the previous theorem for the Hilbert function of $N$, in the following:

Theorem 3.3. Let $C \subseteq \mathbb{P}^{3}$ be a smooth connected curve not lying on any surface of degree smaller than $s$. Then for all $j$ with $\alpha \leq j \leq d-2$, if $t$ is any integer satisfying:

$$
\begin{gathered}
t \leq s \\
\left(\begin{array}{c}
t-1 \\
2
\end{array}\right)<j+1-\partial h_{N}(j)<d-\left(\begin{array}{l}
t \\
2
\end{array}\right)
\end{gathered}
$$

one has:

$$
\partial h_{N}(j+1) \leq \partial h_{N}(j)-t+1 \text {. }
$$

Proof. The claim follows easily by (6) and Theorem 3.2 .

We want to explain roughly how one can use Theorem 3.3 to understand the behavior of $\partial h_{N}(j)$, for $j \in[\alpha+1, d-3]$. Indeed $\alpha$ is the maximum for the function $\partial h_{N}$, achieved for $j=\alpha-1$; in the next few steps, when $j$ is a little bit bigger than $\alpha$, then the difference $j+1-\partial h_{N}(j)$ can be small and one may apply theorem 3.3 only for small (but nevertheless increasing) $t$; so the rate of the descent of $\partial h_{N}$ is small, but increasing. Eventually $j+1-\partial h_{N}(j)$ becomes bigger than $\left(\begin{array}{c}s-1 \\ 2\end{array}\right)$, and one is allowed to apply the theorem taking $s=t$ (of course provided that $j+1-\partial h_{N}(j)$ is still smaller than $d-\left(\begin{array}{l}s \\ 2\end{array}\right)$ ): here $\partial h_{N}(j)$ is forced to decrease by $s-1$ (at least) at any steps. Finally when $j$ approaches $d-2$, then $j+1-\partial h_{N}(j)$ becomes bigger than $d-\left(\begin{array}{c}s \\ 2\end{array}\right)$ and once again one must take $t<s$, in order to apply the theorem: in other words the rate of the descent of $\partial h_{N}$ is allowed to become smoother.

The following picture synthesizes the previous discussion on the shape of $\partial h_{N}$ : 


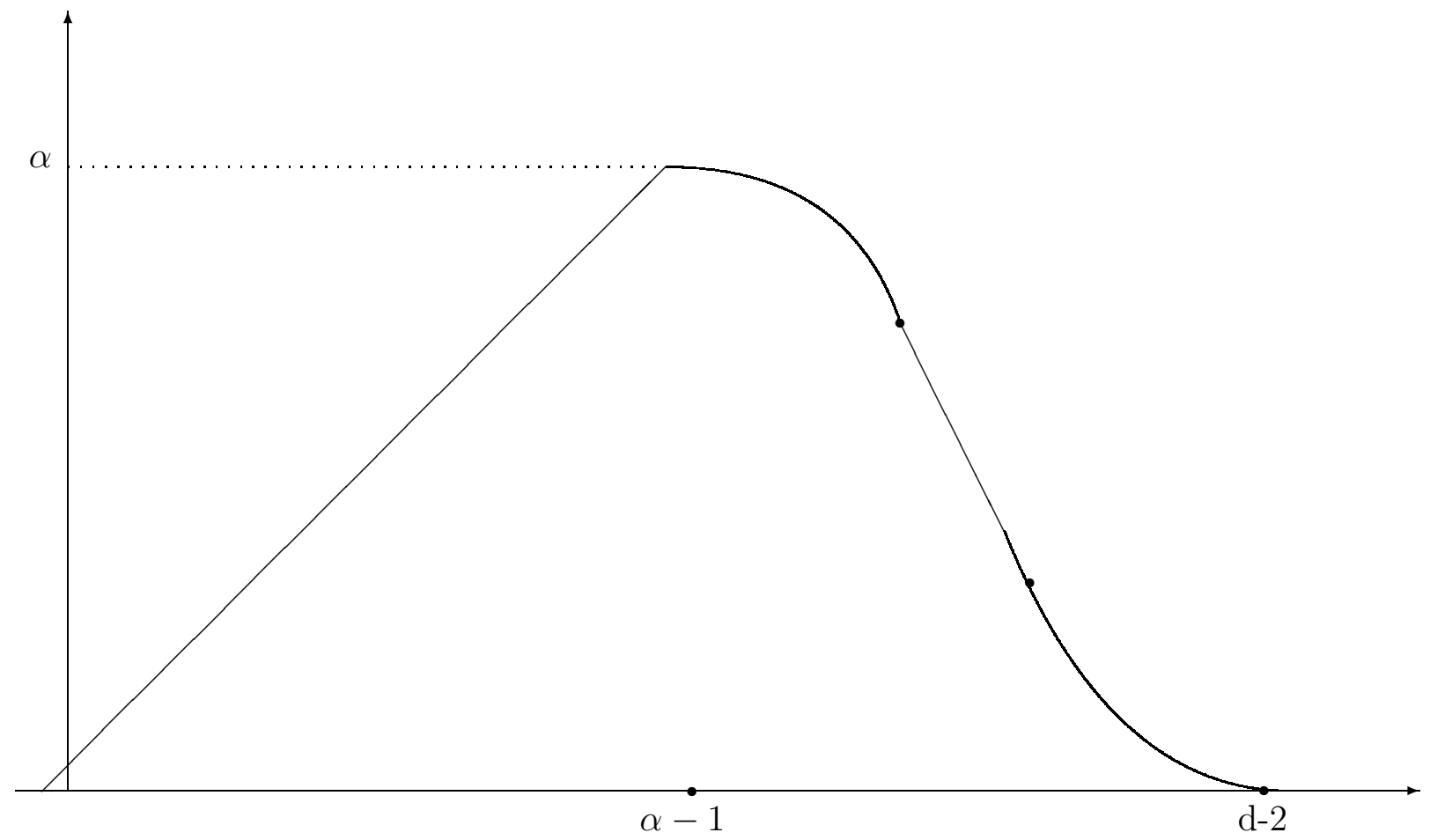

Example 3.4. Let us see the minimal descent of $\partial h_{N}$ in the following numerical case: $d=24, s=4, g=73, \alpha=15$ (here $\delta=180$ ).

The function $\partial h_{N}$ increases by 1 at any step from 0 to $\alpha-1=14$. Then:

- for $j=15$, we get $15=\alpha \geq \partial h_{N}(15)$. Assume the maximum is attained; then for $t=2$, one sees that the inequalities (a),(b) of the previous theorem are satisfied (in fact $t=2$ is the maximum which satisfies the left hand side) so $\partial h_{N}$ is forced to decrease at least by 1 ;

- for $j=16$, we get then $14 \geq \partial h_{N}(16)$. Assume the maximum is attained; then for $t=3$, one sees that the inequalities (a),(b) of the previous theorem are satisfied (in fact $t=3$ is the maximum which satisfies the left hand side) so $\partial h_{N}$ is forced to decrease at least by 2 ;

- for $j=17$, we get then $12 \geq \partial h_{N}(17)$. Assume the maximum is attained; then for $t=4$, one sees that the inequalities (a),(b) of the previous theorem are satisfied (in fact $t=4$ is the maximum which satisfies the left hand side) so $\partial h_{N}$ is forced to decrease at least by 3 ;

- for $j=18$, we get then $9 \geq \partial h_{N}(18)$. Assume the maximum is attained; then the inequality (b) of the previous theorem holds for $t=5$, but $5>s$; we may just conclude that $\partial h_{N}$ is forced to decrease at least by 3 ;

- for $j=19$, we get then $6 \geq \partial h_{N}(19)$. Assume the maximum is attained; then for $t=4$, one sees that the inequalities (a),(b) of the previous theorem are satisfied (in fact, now $t=4$ is the maximum which satisfies the right hand side) so $\partial h_{N}$ is forced to decrease at least by 3 ; 
- for $j=20$, we get then $3 \geq \partial h_{N}(20)$. Assume the maximum is attained; then for $t=3$, one sees that the inequalities (a), (b) of the previous theorem are satisfied (in fact $t=3$ is the maximum which satisfies the right hand side) so $\partial h_{N}$ is forced to decrease at least by 2 ;

- for $j=21$, we get then $1 \geq \partial h_{N}(21)$. Here the maximum must be attained, for $21=d-3$ and we know that $\partial h_{N}(d-3) \geq 1$ for all curves in $\mathbb{P}^{3}$.

Finally, if the maximum is attained in all steps, the function $\partial h_{N}$ reads:

$$
\ldots, 0,1,2,3,4,5,6,7,8,9,10,11,12,13,14,15,15,14,12,9,6,3,1,0, \ldots
$$

and the sum of this function is exactly $180=\delta$, as it should be for the Hilbert function of nodes of a concrete curve.

A similar conclusion holds taking the reverse point of view and using (8) instead of (66).

Theorem 3.5. Let $C \subseteq \mathbb{P}^{3}$ be a smooth connected curve not lying on any surface of degree smaller than $s$. Then for all $j$ with $\alpha \leq j \leq d-2$, if $t$ is any integer satisfying:

$$
\left(\begin{array}{c}
t-1 \\
2
\end{array}\right)<d-j-1+\partial h_{N}(j)<d-\left(\begin{array}{l}
t \\
2
\end{array}\right)
$$

one has:

$$
\partial h_{N}(j-1) \geq \partial h_{N}(j)+t-1 .
$$

Proof. The claim follows from (18) and Theorem 3.2

Example 3.6. Let us take the previous numerical example, starting this time from $j=$ $d-3$ and going backwards; assume still $d=24, s=4, g=73, \alpha=15$ (here $\delta=180$ ).

The function $\partial h_{N}$ increases by 1 at any step from 0 to $\alpha-1=14$, so that also $\partial h_{N}(14)=15$.

- for $j=21=d-3$, we must have $\partial h_{N}(21) \geq 1$. Assume that the minimum is attained; then for $t=3$, one sees that the inequalities (a),(b) of Theorem 3.5] are satisfied (in fact $t=3$ is the maximum which satisfies the left hand side) so $\partial h_{N}$ is forced to increase at least by $t-1=2$, going one step back;

- for $j=20$, we get then $\partial h_{N}(20) \geq 3$. Assume the minimum is attained; then for $t=4$, one sees that the inequalities (a),(b) of the previous theorem are satisfied (in fact $t=4$ is the maximum which satisfies the left hand side) so $\partial h_{N}$ is forced to increase at least by 3 , going one step back;

- for $j=19$, we get then $\partial h_{N}(19) \geq 6$. Assume the minimum is attained; then for $t=5$, one sees that condition (b) of the previous theorem is satisfied, but condition (a) fails. All we can say, taking again $t=4$, is that $\partial h_{N}$ is forced to increase at least by 3 , going one step back;

- for $j=18$, we get then $\partial h_{N}(18) \geq 9$. Assume the minimum is attained; then for $t=5$, one sees that condition (b) of the previous theorem is satisfied, but condition (a) fails. All we can say, taking again $t=4$, is that $\partial h_{N}$ is forced to increase at least by 3 , going one step back; 
- for $j=17$, we get then $\partial h_{N}(18) \geq 12$. Assume the minimum is attained; then for $t=3$, one sees that the inequalities $(\mathrm{a}),(\mathrm{b})$ of the previous theorem are satisfied (in fact, now $t=3$ is the maximum which satisfies the right hand side) so $\partial h_{N}$ is forced to increase at least by 2 , going one step back;

- for $j=16$, we get then $\partial h_{N}(16) \geq 14$. Assume the minimum is attained; then for $t=2$, one sees that the inequalities (a),(b) of the previous theorem are satisfied (in fact, now $t=3$ is the maximum which satisfies the right hand side) so $\partial h_{N}$ is forced to increase at least by 1 , going one step back;

- for $j=15=\alpha$, finally we get then $\partial h_{N}(21) \geq 15=\alpha$. Here the minimum must be attained.

Finally, if the minimum is attained in all steps, the function $\partial h_{N}$ reads:

$$
\ldots, 0,1,2,3,4,5,6,7,8,9,10,11,12,13,14,15,15,14,12,9,6,3,1,0, \ldots
$$

which are exactly the same values as before.

Notice that the numbers $d=24, s=4, g=73, \alpha=15$ above are not random: they are the numbers of a complete intersection $C$ of type $(4,6)$, see Example 1.7

\section{Minimal node functions And Halphen CURVES}

In this section we use the previous results to construct a new function which bounds $\partial h_{N}$ from below, and we show that this bound is sharp for the so-called Halphen's curves (see Theorem 4.10). Further examples will be given in the next section.

Definition 4.1. (a) Given integers $d, s, n$ with $d \geq 3, s \geq 2$ and $n \geq 3$ we define a numerical function $\bar{\Delta}$ in the interval $[0, d-3]$ by descending induction (motivated by Theorems 1.3 and 3.5 ) as follows: (1) $\bar{\Delta}(d-3):=n-2$

(2) for $d-4 \geq j \geq 0$,

$$
\bar{\Delta}(j-1):=\bar{\Delta}(j)+\max \{t-1, n-2\}
$$

where

$$
t:=\max \left\{i \leq s \mid\left(\begin{array}{c}
i-1 \\
2
\end{array}\right)<d-j-1+\bar{\Delta}(j)<d-\left(\begin{array}{l}
i \\
2
\end{array}\right)\right\}
$$

(we agree that the maximum of the empty set is $-\infty$ ).

(b) Given integers $d, s, n, \alpha$ with $d \geq 3, s \geq 2, n \geq 3$ and $\alpha \geq 0$ we define the minimal node function $\Delta$ with respect to $d, s, n, \alpha$ as follows:

$$
\Delta(j):=\left\{\begin{array}{ll}
0 & \text { if } \quad j<0 \quad \text { or } \quad j>d-3 \\
j+1 & \text { if } \quad 0 \leq j \leq \alpha-1 \\
\bar{\Delta}(j) & \text { if } \quad \alpha \leq j \leq d-3
\end{array} .\right.
$$

We set

$$
\delta(\Delta):=\sum_{j=0}^{\infty} \Delta(j)
$$


The next theorem shows that minimal node functions provide lower bounds for $\partial h_{N}$, which are sharp in some significant cases, as we shall see later.

Theorem 4.2. Let $C \subseteq \mathbb{P}^{3}$ be a smooth connected curve of degree d, lying on no surface of degree smaller than $s(s \geq 2)$. Let $\Delta$ be the minimal node function, with respect to $d$, $s, n, \alpha$. Then $\partial h_{N}(j) \geq \Delta(j)$ for all $j \in \mathbb{Z}$.

Proof. Notice that $\partial h_{N}(j)=\Delta(j)$ for all $j$ outside of the interval $[\alpha, d-4]$, and in particular $\partial h_{N}(d-3)=\Delta(d-3)=n-2$. We prove the non-trivial part of our statement by descending induction on $j$, starting with $j=d-3$; namely we show that, for $\alpha+1 \leq$ $j \leq d-3, \partial h_{N}(j) \geq \Delta(j)$ implies $\partial h_{N}(j-1) \geq \Delta(j-1)$.

Assume then that $\partial h_{N}(j) \geq \Delta(j)$ for some $j$ with $d-3 \geq j \geq \alpha+1$. Set

$$
t:=\max \left\{i \leq s \mid\left(\begin{array}{c}
i-1 \\
2
\end{array}\right)<d-j-1+\Delta(j)<d-\left(\begin{array}{l}
i \\
2
\end{array}\right)\right\}
$$

and similarly

$$
t^{\prime}:=\max \left\{i \leq s \mid\left(\begin{array}{c}
i-1 \\
2
\end{array}\right)<d-j-1+\partial h_{N}(j)<d-\left(\begin{array}{l}
i \\
2
\end{array}\right)\right\} .
$$

We observe that $t^{\prime} \geq 1$. Indeed $d-j-1+\partial h_{N}(j)<d-\left(\begin{array}{l}1 \\ 2\end{array}\right)=d$, because $\partial h_{N}(j) \leq$ $\alpha<j+1$, and $d-j-1+\partial h_{N}(j)>\left(\begin{array}{l}0 \\ 2\end{array}\right)=0$ because $\partial h_{N}(j)>0$.

If $t-1 \leq n-2$ we have $\Delta(j-1)=\Delta(j)+n-2$, and since $\partial h_{N}(j-1) \geq \partial h_{N}(j)+n-2$ by Theorem 1.3, the conclusion is clear.

If $t-1>n-2$ we argue by contradiction, assuming that $\partial h_{N}(j-1)<\Delta(j-1)$. Since $\Delta(j-1)=\Delta(j)+t-1$ by definition, while $\partial h_{N}(j-1) \geq \partial h_{N}(j)+t^{\prime}-1$ by Theorem 3.5. the above inequality and the induction assumption imply

$$
t-t^{\prime}>\partial h_{N}(j)-\Delta(j) \geq 0
$$

whence necessarily $t^{\prime}+1 \leq t \leq s$. Now the definition of $t^{\prime}$ guarantees that at least one of the following inequalities holds:

$$
\begin{gathered}
\left(\begin{array}{c}
t^{\prime} \\
2
\end{array}\right) \geq d-j-1+\partial h_{N}(j) \\
d-j-1+\partial h_{N}(j) \geq d-\left(\begin{array}{c}
t^{\prime}+1 \\
2
\end{array}\right)
\end{gathered}
$$

On the other hand by the definition of $t$, the inequality $t^{\prime} \leq t-1$ and the induction hypothesis we have:

$$
\left(\begin{array}{l}
t^{\prime} \\
2
\end{array}\right)<d-j-1+\Delta(j) \leq d-j-1+\partial h_{N}(j)
$$

whence (10) is false and (11) must be true.

Set $u:=t-t^{\prime}-1 \geq 0$. Then by (11) we have:

$$
\partial h_{N}(j) \geq j+1-\left(\begin{array}{c}
t^{\prime}+1 \\
2
\end{array}\right)
$$


while

$$
-\Delta(j)>-j-1+\left(\begin{array}{l}
t \\
2
\end{array}\right)
$$

by definition. Then by (9) we get

$$
u \geq \partial h_{N}(j)-\Delta(j)>\left(\begin{array}{l}
t \\
2
\end{array}\right)-\left(\begin{array}{c}
t^{\prime}+1 \\
2
\end{array}\right)=\frac{\left(2 t^{\prime}+1\right) u+u^{2}}{2} \geq 0
$$

whence $u>0$ and $1>2 t^{\prime}+u$. This is a contradiction because $t^{\prime}>0$.

In view of Theorem 4.2 it is natural to give the following Definition.

Definition 4.3. Let $C \subseteq \mathbb{P}^{3}$ be a curve of degree $d$ not lying on any surfaces of degree $s(s \geq 2)$. We say that $C$ has minimal nodal postulation when $\partial h_{N}$ coincides with the minimal node function with respect to $d, s, n, \alpha$.

From the above theorem we get the following two Corollaries which give an upper bound for the genus and a lower bound for $\alpha$ (whence an upper bound for the speciality). These bounds are sharp in some important cases (see Proposition 4.8. Theorem 4.10 and section [5]).

Corollary 4.4. Let the notation and the assumptions be as in Theorem 4.2. Then:

(i) $\delta \geq \delta(\Delta)$ and equality holds if and only if $C$ has minimal node postulation;

(ii) $g \leq \frac{(d-1)(d-2)}{2}-\delta(\Delta)$ and equality holds if and only if $C$ has minimal nodal postulation.

Proof. It is an immediate consequence of Theorem 4.2 .

Corollary 4.5. Let the notation and the assumptions be as in Theorem 4.2 and let $\bar{\Delta}$ be as in Definition 4.1. Set

$$
\bar{\alpha}:=\min \{j \geq 0 \mid \bar{\Delta}(j) \leq j\} .
$$

Then $\alpha \geq \bar{\alpha}$ and $e \leq d-3-\bar{\alpha}$.

Proof. Let $\Delta$ be the minimal node function with respect to $d, s, n$ and $\alpha$. Then by Theorem 4.2 and Proposition 1.2 we have $\Delta(\alpha) \leq \partial h_{N}(\alpha) \leq \alpha$, whence the conclusion by the definition of $\Delta$.

Remark 4.6. (i) Let us observe that once we fix $d, s n$ and $\alpha$, the minimal nodal function $\Delta$ with respect to these values is completely determined, so is its sum $\delta(\Delta)$. Hence the upper bound for $g$ given by Corollary 4.4 depends only on these numbers, or, equivalently, on $s, d n$ and $e$.

Likewise if we fix $d, s$ and $n$ the lower bound $\bar{\alpha}$ for $\alpha$ given by Corollary 4.5 is completely determined by $d$ and $s$.

(ii) We will be able to compute explicitly $\bar{\alpha}$ under the assumption $d>s(s-1)$ and $n=3$ (see Proposition 4.8), and from this we will be able to classify the curves with minimal nodal postulation and minimal $\alpha$ in the given range: they are, of course, the well-known Halphen curves (see Theorem 4.10). 
The study of curves with minimal nodal postulation but non-minimal $\alpha$ seems to be much harder; in particular it is not clear whether the above bound for the genus is sharp. We will give some partial results for $d>s(s-1)$ and $s \leq 4$ (see Section 5 ).

Our results and examples seem to indicate that the classification of curves with minimal nodal postulation is not straightforward and could be a real challenge.

Before coming to the main results of this section we want to say something on complete intersections. Observe that our previous examples 3.4 and 3.6 show that complete intersection curves of type $(4,6)$ have minimal nodal postulation. More generally the following example shows that this is true for all complete intersections.

Example 4.7. Let $C \subseteq \mathbb{P}^{3}$ be a complete intersection of type $(s, s+u)$, with $s \geq 2$ and $u \geq 0$. Then $d=s^{2}+s u, s$ has the usual meaning, $\alpha=d-2 s-u+1$ (see Example 1.7). Moreover $\partial h_{N}(d-3)=1$, whence $n=3$. The minimal nodal function $\Delta$ with respect to $d, \alpha, s$ as above and $n=3$ can be computed easily from the definition and is:

$$
\Delta(j)= \begin{cases}0 & \text { if } j<0 \\
j+1 & \text { if } 0 \leq j<\alpha \\
\alpha-\left(\begin{array}{c}
j-\alpha+1 \\
2
\end{array}\right) & \text { if } \alpha \leq j \leq d-s-u-2=\alpha+s-3 \\
\left(\begin{array}{l}
s \\
2
\end{array}\right)+(d-s-1-j)(s-1) & \text { if } d-s-u-1 \leq j \leq d-s-2 \\
\left(\begin{array}{c}
d-1-j \\
2
\end{array}\right) & \text { if } d-s-1 \leq j \leq d-3 \\
0 & \text { if } j>d-3\end{cases}
$$

By Example 1.7 we have $\Delta=\partial h_{N}$, that is $C$ has minimal nodal postulation.

Now we want to show that minimal nodal postulation is achieved not only by complete intersection curves but also by Halphen's curves. We begin by showing a lower bound for $\alpha$. This bound is well-known, but we give a new proof as a consequence of our theory of minimal nodal functions.

Proposition 4.8. Let $C \subseteq \mathbb{P}^{3}$ be a curve of degree $d$ not lying on any surface of degree $<s$. Assume $s \geq 2$ and $d>s(s-1)$. Let $(k, u)$ be the unique pair of integers such that $d=s^{2}+u s-k, 0 \leq k \leq s-1$ and $u \geq 0$. Assume further that $C$ is not a complete intersection (i.e. $k \geq 1$ ). Then we have:

(i) $\bar{\alpha} \geq d-2 s-u+2$, whence $\alpha \geq d-2 s-u+2$ (where $\bar{\alpha}$ is defined in Corollary 4.5);

(ii) if $\alpha=d-2 s-u+2$, then the minimal nodal function with respect to $d, s, \alpha$ and $n=3$ is the following:

$$
\Delta(j)= \begin{cases}0 & \text { if } j<0 \\
j+1 & \text { if } 0 \leq j<\alpha \\
\alpha-\left(\begin{array}{c}
j-\alpha+1 \\
2
\end{array}\right) & \text { if } \alpha \leq j \leq \alpha+k-2 \\
\alpha-\left(\begin{array}{c}
j-\alpha+1 \\
2
\end{array}\right)-(j+2-\alpha-k) & \text { if } \alpha+k-1 \leq j \leq \alpha+s-4=d-s-u-2 \\
\left(\begin{array}{l}
s \\
2
\end{array}\right)+(d-s-1-j)(s-1) & \text { if } d-s-u-1 \leq j \leq d-s-2 \\
\left(\begin{array}{c}
d-1-j \\
2
\end{array}\right) & \text { if } d-s-1 \leq j \leq d-3 \\
0 & \text { if } j>d-3\end{cases}
$$


Proof. To prove (i) we use Corollary 4.5. A lengthy and tedious but elementary calculation allows to compute the function $\bar{\Delta}$ (see Definition 4.1) with respect to $d, s$ and $n=3$. Going backwards step by step starting with $d-3$ and using repeatedly the assumption $d>s(s-1)$ we get:

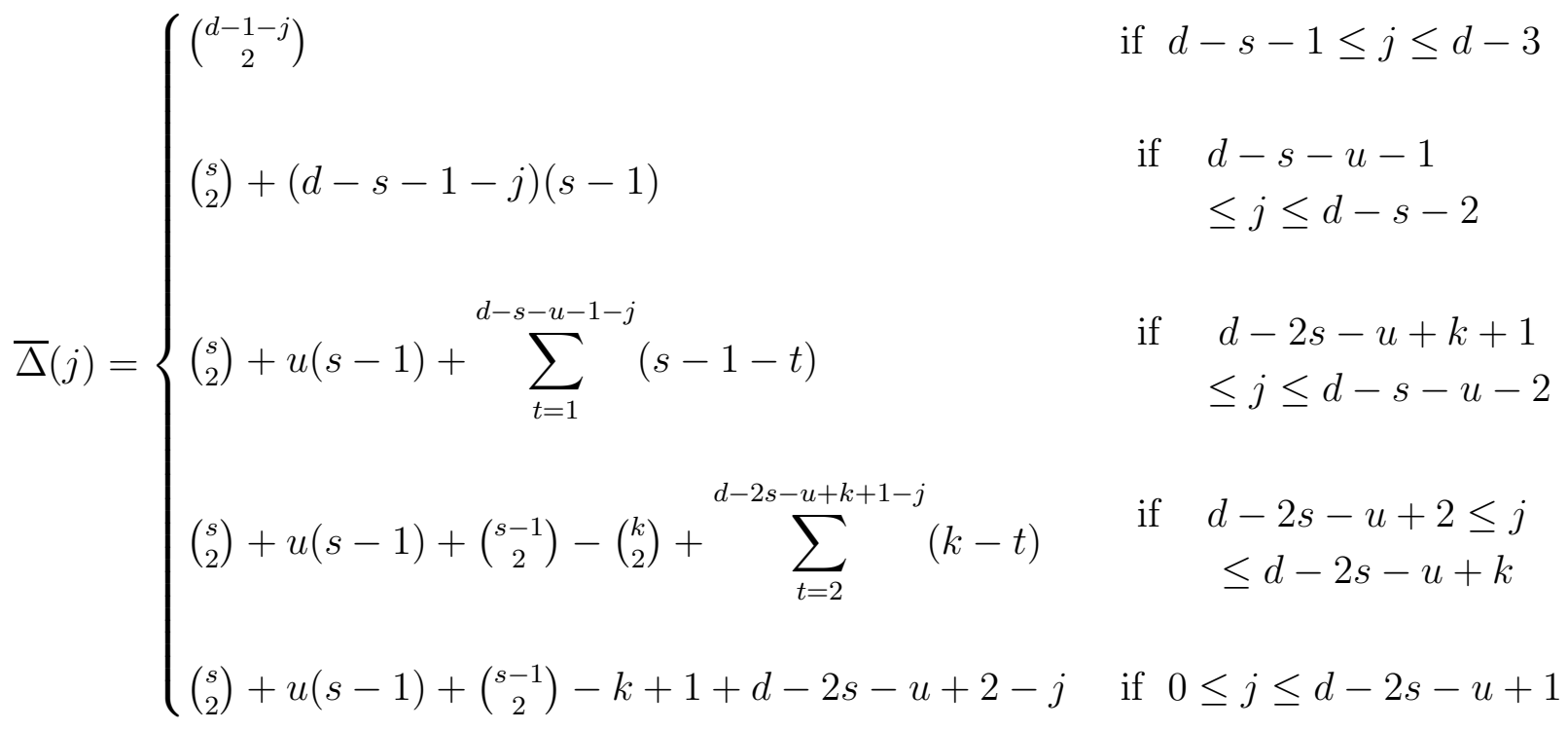

If $k \geq 2$ we have $\bar{\Delta}(d-2 s-u+2)=d-2 s-u+2$, and if $k=1$ we have $\bar{\Delta}(d-2 s-u+2)=$ $d-2 s-u+1$. Then (i) follows from Corollary 4.5.

The proof of (ii) is another tedious but elementary calculation using (i) and the definition of $\Delta$.

Remark 4.9. Since $e=d-3-\alpha$ it is immediate to see that the bound of Proposition 4.5 is equivalent to the Halphen-Gruson-Peskine bound $e \leq \frac{d}{s}+s-4$. The first modern proof of this bound for $e$ is in 11 .

Another proof of the bound for $e$ can be found in [16], where the Halphen-GrusonPeskine bound is generalized to non-integral curves, using a fine study of the spectrum.

Theorem 4.10. Let $C \subseteq \mathbb{P}^{3}$ be a curve of degree $d$ not lying on any surface of degree $<s$. Assume $s \geq 2$ and $d>s(s-1)$. Let $(k, u)$ be the unique pair of integers such that $d=s^{2}+u s-k$, with $0 \leq k \leq s-1$ and $u \geq 0$. Then the following are equivalent:

(i) C lies on a surface $S$ of degree $s$ and it is either a complete intersection of $S$ with a surface of degree $s+u($ if $k=0)$, or it is linked on $S$ to a plane curve of degree $k$, if $k>0$; 
(ii) if $k=0$ the function $\partial h_{N}$ is as in Example 4.7. If $k>0$ we have $\alpha=d-2 s-u+2$ and

$$
\partial h_{N}(j)= \begin{cases}0 & \text { if } j<0 \\
j+1 & \text { if } 0 \leq j<\alpha \\
\alpha-\left(\begin{array}{c}
j-\alpha+1 \\
2
\end{array}\right) & \text { if } \alpha \leq j \leq \alpha+k-2 \\
\alpha-\left(\begin{array}{c}
j-\alpha+1 \\
2
\end{array}\right)-(j+2-\alpha-k) & \text { if } \alpha+k-1 \leq j \leq \alpha+s-4=d-s-u-2 ; \\
\left(\begin{array}{l}
s \\
2
\end{array}\right)+(d-s-1-j)(s-1) & \text { if } d-s-u-1 \leq j \leq d-s-2 \\
\left(\begin{array}{c}
d-1-j \\
2
\end{array}\right) & \text { if } d-s-1 \leq j \leq d-3 \\
0 & \text { if } j>d-3\end{cases}
$$

(iii) $C$ has minimal nodal postulation with respect to $d, s, n=3$ and

$$
\alpha= \begin{cases}d-2 s-u+2 & \text { if } k>0 \\ d-2 s-u+1 & \text { if } k=0\end{cases}
$$

In other words: if $\Delta$ is the minimal node function with respect to $d s$ and $n=3$ we have:

$$
\Delta(j)=\partial h_{N}(j) \text { for every } j \in \mathbb{Z}
$$

(iv) $C$ has maximal genus in the set of all curves of degree $d$ not lying on any surface of degree $<s$.

Proof. (i) $\Rightarrow$ (ii). If $k=0$ follows immediately by Example 4.7

Assume now $k>0$ and let $Y$ be the plane curve linked to $C$ on $S$. Then $\alpha=d-2 s-u+2$, since $\omega_{C}$ is cut by surfaces of degree $2 s+u-4$ passing through $Y$ (outside the intersection $Y \cap C)$. Moreover $C$ is aCM, and hence to compute $\partial h_{N}$ we can use Example 1.7. Now the general hyperplane section $\Gamma$ of $C$ is directly linked in a complete intersection $(s, s+u)$ with the general hyperplane section of $Y$, which is collinear of degree $k$. Then by a well known relation between the Hilbert functions of linked zero-dimensional schemes (see [10]) we have

$$
\partial h_{\Gamma}(j)= \begin{cases}0 & \text { if } j<0 \\ j+1 & \text { if } 0 \leq j \leq s-1 \\ s & \text { if } s-1 \leq j \leq s+u-1 \\ 2 s+u-1-j & \text { if } s+u \leq j \leq 2 s+u-k-2 \\ 2 s+u-2-j & \text { if } 2 s+u-k-1 \leq j \leq 2 s+u-3 \\ 0 & \text { if } j \geq 2 s+u-2\end{cases}
$$

On the other hand by Example 1.7 we have

$$
\partial^{2} h_{N}(j)=1-\partial h_{\Gamma}(d-1-j)
$$

and a straightforward calculation shows (ii).

(ii) $\Longleftrightarrow$ (iii). If $k=0$ follows from Example 4.7. If $k>0$ use Proposition 4.8,

(iii) $\Longleftrightarrow$ (iv). It is an easy consequence of Theorem 4.2 and Proposition 4.8 .

(ii) $\Rightarrow\left(\right.$ i). Assume first that $k>0$ and let $C^{\prime}$ be a curve of degree $d$ linked to a plane curve of degree $k$ by a complete intersection of type $(s, s+u)$. Let $N^{\prime}$ be the set 
of nodes of a general plane projection of $C^{\prime}$. Then $\partial h_{N}=\partial h_{N^{\prime}}$, whence by Remark 1.1(iii) $h^{0}\left(\mathcal{O}_{C}(j)\right)=h^{0}\left(\mathcal{O}_{C^{\prime}}(j)\right)$ for all $j \in \mathbb{Z}$. This easily implies, since $C^{\prime}$ is aCM, that $h^{0}\left(\mathcal{I}_{C}(j)\right)-h^{0}\left(\mathcal{I}_{C^{\prime}}(j)\right)=h^{1}\left(\mathcal{I}_{C}(j)\right)$, whence, in particular, $h^{0}\left(\mathcal{I}_{C}(j)\right) \geq h^{0}\left(\mathcal{I}_{C^{\prime}}(j)\right)$. By easy degree considerations this implies that $h^{0}\left(\mathcal{I}_{C}(j)\right)=h^{0}\left(\mathcal{I}_{C^{\prime}}(j)\right)$ for $j \leq s+u-1$, whence $h^{1}\left(\mathcal{I}_{C}(j)\right)=0$ for $j \leq s+u-1$. In particular $C$ is contained in an irreducible surface $S$ of degree $s$, and a surface $T$ of degree $s+u$ not containing $S$.

Let now $\Gamma$ and $\Gamma^{\prime}$ be the general hyperplane sections of $C$ and $C^{\prime}$ respectively. Then by Remark 1.6] it follows that $h_{\Gamma^{\prime}}(j) \geq h_{\Gamma}(j)$ for every $j$. We want to show that $h_{\Gamma^{\prime}}=$ $h_{\Gamma}$. Assume this is not true and set $t:=\min \left\{j \in \mathbb{Z} \mid h_{\Gamma^{\prime}}(j)>h_{\Gamma}(j)\right\}$. By the above considerations we have that $t \geq s+u$ and that $\partial h_{\Gamma}$ is strictly decreasing for $j \geq t$ until it vanishes. Now $\partial h_{\Gamma^{\prime}}$ is explicitly given in (12) (with $\Gamma$ replaced by $\Gamma^{\prime}$ ), and since $\sum \partial h_{\Gamma^{\prime}}(j)=d=\sum \partial h_{\Gamma}(j)$ an easy calculation leads to a contradiction.

Then $h_{\Gamma^{\prime}}=h_{\Gamma}$, whence $C$ is aCM by Remark 1.6 and $\Gamma$ is linked to a collinear scheme in a complete intersection of type $(s, s+u)$. Then it is easy to see that $S \cap T=C \cup C^{\prime \prime}$, where $C^{\prime \prime}$ is a plane curve of degree $k$.

If $k=0$ the proof is similar to (and easier than) the previous one and is left to the reader.

Example 4.11. There are some (few) examples in which the minimal node function is the effective Hilbert function of the nodes of a curve coming from $\mathbb{P}^{r}, r \geq 4$. This is the case, for instance, of a curve $C \subseteq \mathbb{P}^{3}$ which is a general projection of a Castelnuovo curve in $\mathbb{P}^{r}$, at least when $d>(r-2)(r-1)$.

Indeed every Castelnuovo curve $C^{\prime} \subseteq \mathbb{P}^{r}$ with $d>2 r$ lies on a surface of (minimal) degree $r-1$ (see [3] or [12]), which is also the minimal degree of a surface containing the projection $C$ of $C^{\prime}$ to $\mathbb{P}^{3}$, if $d>(r-2)(r-1)$. Then in the definition of minimal node function, we get here $t \leq s=r-1$ so that $\max \{t-1, r-2\}=r-2$ hence, by construction $4.1(2)$ ), the minimal node function satisfies:

$$
\Delta(j-1)=\Delta(j)+r-2 \text { for } \quad \alpha \leq j \leq d-3 .
$$

Now just observe that Castelnuovo's curves reach exactly this bound by Proposition [2.1]

In order to get more refined bounds, similar to the ones in $\mathbb{P}^{3}$, it seems reasonable to take into account some flag conditions, such as, for example, the ones used in [6] to bound the genus.

Remark 4.12. The curves of the previous example are the first examples of non-aCM curves attaining the lower bound stated in Theorem 4.2. Indeed they are not even linearly normal.

Observe also that for these curves we have $\alpha=\left\lceil\frac{(d-2)(r-2)}{r-1}\right\rceil$, whence $\alpha$ is not minimal with respect to $d$ and $s=r-1>2$.

We will see in the next section that if $\alpha$ is not minimal there are also linearly normal non-aCM curves having minimal node postulation. 


\section{Curves on surfaces of SMall Degree}

In this section we study a class of curves having minimal node postulation, with $n=3$, $s \leq 4, d>s(s-1)$ and $\alpha$ not minimal. This shows, in particular, that the Halphen type bound for the genus in terms of $d, s$ and $e$ given in Corollary 4.4) is sharp in some non-trivial cases. We leave the easy translation to the reader.

5.1. The case $s=2$. If $s=2$ the inequality $d>s(s-1)$ is always satisfied, the minimal node function $\Delta$ coincides with the lower bound given by Theorem 1.3 and is defined as follows:

$$
\Delta(j)=\left\{\begin{array}{ll}
0 & \text { if } j<0 \\
j+1 & \text { if } 0 \leq j \leq \alpha-1 \\
d-2-j & \text { if } \alpha \leq j \leq d-3 \\
0 & \text { if } j \geq d-2
\end{array} .\right.
$$

It follows that

$$
\delta(\Delta)=\frac{1}{2} \alpha(\alpha+1)+\frac{1}{2}(d-2-\alpha)(d-1-\alpha) .
$$

Proposition 5.1. Assume $C$ lies on a quadric surface $Q$. Then:

(a) if $Q$ is smooth and $C$ is of type $(a, b)$ with $a \geq b$, then $\alpha=a-1$;

(b) if $Q$ is a cone then

$$
\alpha=\left\{\begin{array}{ll}
\frac{d}{2}-1 & \text { if } d \text { is even } \\
\frac{d-1}{2} & \text { if } d \text { is odd }
\end{array} ;\right.
$$

(c) C has minimal node postulation (4.3).

Proof. (a) We have $e=b-2$ (e.g. by the adjunction formula) and the conclusion follows by Remark 1.1.

(b) If $d$ is even $C$ is a complete intersection of type $\left(2, \frac{d}{2}\right)$ and then $e=\frac{d}{2}-2$, whence $\alpha=\frac{d}{2}-1$.

If $d$ is odd then $C$ is linked to a line $\ell$ in a complete intersection of type $\left(2, \frac{d+1}{2}\right)$. Then the complete canonical series of $C$ is cut out by the linear system of all surfaces of degree $\frac{d+1}{2}-2$ containing $\ell$, outside the divisor $\ell \cdot C$. It follows that $e=\frac{d+1}{2}-3$, whence $\alpha=\frac{d-1}{2}$.

(c) Set $p:=d-2-\alpha$. Then $p \geq 0$ and a straightforward calculation, using (a), (b) and (14) shows that

$$
\delta=\frac{1}{2} \alpha(\alpha+1)+\frac{1}{2} p(p+1)=\delta(\Delta)
$$

The conclusion follows by Theorem 1.3 and an easy computation.

The following Proposition gives a characterization of curves lying on a quadric surfaces, which completes Proposition 5.1

Proposition 5.2. Let $C \subseteq \mathbb{P}^{r}, r \geq 3$, be a non degenerate curve. Then the following are equivalent:

(a) $r=3, C$ is special and is contained in a quadric;

(b) $\alpha \leq d-4$ and $C$ has minimal node postulation (13); 
(c) $d \geq 6$ and $\partial h_{N}(d-4)=2$;

(d) $C$ is special and $\partial h_{N}(d-4)=2$.

Proof. (a) $\Rightarrow$ (b) follows from Remark 1.1(v) and Proposition 5.1 .

(b) $\Rightarrow$ (c). Since $\alpha \leq d-4$ it is immediate that $\partial h_{N}(d-4)=2$. Moreover $d-4 \geq$ $\alpha \geq \partial h_{N}(d-4)=2$, whence $d \geq 6$.

(c) $\Rightarrow$ (d). Assume that $C$ is non-special. Then by Remark 1.1(v) we have either $\alpha=d-3$ or $\alpha=d-2$. In the first case we have $d-3=\partial h_{N}(d-4)=2$, whence $d=5$, a contradiction. In the second case we have, by Proposition 1.2, $d-2=\partial h_{N}(d-3)=$ $\partial h_{N}(d-4)+1=3$, whence $d=5$, again a contradiction.

(d) $\Rightarrow$ (a). Since $C$ is special we have $\alpha \leq d-4$. Moreover by Theorem 1.3 we have $0<\partial h_{N}(d-3)<\partial h_{N}(d-4)=2$, whence $\partial h_{N}(d-3)=1$. This implies $r=3$.

Assume now $d \geq 7$. If $C$ is not contained in a quadric we can apply Theorem 3.5 with $t=s=3$ and $j=d-3$. This implies that $\partial h_{N}(d-4) \geq \partial h_{N}(d-3)+t-1=3$, a contradiction.

Finally assume $d=6$. Then $d-3=3$ and we must have $\partial h_{N}: \ldots, 0,1,2,2,1,0, \ldots$ Then by [7, Proposition 5.5, $C$ is a complete intersection of type $(2,3)$, and the conclusion follows also in this case.

As an application of the above Proposition we give the following characterization of the curves in $\mathbb{P}^{3}$ having minimal $\alpha$.

Proposition 5.3. Assume $C \subseteq \mathbb{P}^{3}$. Then $\alpha=\left\lceil\frac{d-2}{2}\right\rceil$ (that is $\alpha$ is minimal, see Corollary 1.4) if and only if one (and only one) of the following holds:

(i) $(d, g) \in\{(3,0),(4,1),(5,1),(5,2)\}$ (in particular $C$ is non-special);

(ii) $C$ is a special Castelnuovo curve;

(iii) $d=9$ and $C$ is a complete intersection of two cubics;

(iv) $d=7$ and $C$ is linked to a conic in a complete intersection of type $(3,3)$.

Proof. Assume (i). Then $\alpha=d-2=1$ if $(d, g)=(3,0)$ and $\alpha=d-3$ in the remaining cases. Then $\alpha=\left\lceil\frac{d-2}{2}\right\rceil$.

If (ii) holds then $\alpha$ is minimal by Proposition 5.1 (see also remark 5.4)

If either (iii) or (iv) hold then $\alpha$ is minimal by a direct calculation (see e.g. Example 4.7 and Theorem 4.10).

Assume now that $\alpha=\left\lceil\frac{d-2}{2}\right\rceil$.

If $C$ is non-special then $d-3 \leq \alpha \leq d-2$. If $\alpha=d-2$ then $d=3$ and $g=0$. If $\alpha=d-3$ we have either $d=4$ or $d=5$. In the first case $\alpha=1$ and $\partial h_{N}: \ldots 0,1,1,0 \ldots$, whence $g=1$. In the second case $\alpha=2$ and either $\partial h_{N}: \ldots 0,1,2,1,0 \ldots$ (whence $g=1$ ); or $\partial h_{N}: \ldots 0,1,2,2,0 \ldots$ (whence $g=2$ ).

If $C$ is special and lies on a quadric then $C$ is a Castelnuovo curve by Proposition 5.1.

Finally assume that $C$ is special and does not lie on a quadric. Then $d \geq 6$ and by [7, Proposition $5.5 d$ must be odd. Then by Proposition 4.8 (with $s=3$ ) we must have either $d=9$ or $d=7$. Moreover by Proposition 5.2 we must have

$$
\partial h_{N}(d-4)>2 .
$$


Assume $d=9$, whence $\alpha=4<d-4$. Then by Theorem 1.3 and (15) we must have $\partial h_{N}(d-4)=3$ and $\partial h_{N}(d-3)=1$. It follows

$$
\partial h_{N}: \ldots 0,1,2,3,4,4,3,1,0 \ldots,
$$

and then $C$ is a complete intersection of type $(3,3)$ by Theorem 4.10 (with $d=9, s=3$, $u=k=0)$.

Assume finally that $d=7$. Then an argument as the previous one shows that

$$
\partial h_{N}: \ldots 0,1,2,3,3,1,0 \ldots
$$

Then $C$ is linked to a conic in a complete intersection of type $(3,3)$, again by Theorem 4.10 (with $d=7, s=3, u=0, k=2$ ).

Remark 5.4. (i) Proposition 5.1 (c) shows that every curve in $\mathbb{P}^{3}$ lying on a quadric, has minimal node postulation. This is true in particular also by non-Castelnuovo curves (that is curves of type $(a, b)$ on a smooth quadric, with $|a-b| \geq 2)$.

Moreover it is easy to check that every function (13) occurs on a smooth quadric, provided $\alpha$ satisfies the obvious requirement $\alpha \geq d-2-\alpha$ (just take curves of type $(\alpha+1, d-\alpha-1)$ on the quadric).

(ii) On the other hand Proposition 5.2 shows that every special curve with minimal node postulation (13) must lie on a quadric, whence by Proposition 5.3 every special curve in $\mathbb{P}^{3}$ achieving both the bound of Theorem 1.3 and the lower bound for $\alpha$ is necessarily a Castelnuovo curve.

(iii) It follows easily by Remark 1.1(v) that a non-special curve $C \subseteq \mathbb{P}^{3}$ has $\alpha \geq d-3$ hence it trivially attains the lower bound of Theorem 1.3 , by Riemann-Roch and by 1.1 (iii). On the other hand such a $C$ does not necessarily lie on a quadric surface (e.g. general rational curves of degree $d \geq 5$ and elliptic curves of degree $d \geq 5$ ). Compare with Proposition 5.2.

(iv) We feel it would be interesting to generalize the above results to higher-dimensional spaces, replacing quadric surfaces with surfaces of minimal degree. In particular we don't know if (ii) holds for such curves.

5.2. The case $s=3$. The case where $\alpha$ is minimal and $C$ has the minimal node function is yet covered by Theorem 4.10 (compare condition (ii)). Hence we may assume that $\alpha$ is not minimal. It is easy to see that the minimal node function can be written as follows, for suitable integers $v \geq 0$ and $z \geq-1$ :

$$
\Delta: \ldots, 0,1, \ldots, v+2 z+1,2 z+1, \ldots, 3,1,0, \ldots
$$

Here $d=v+3 z+4, \alpha=2 z+v+1$ and if $v=0$ the value $2 z+1$ is repeated twice, that is $\Delta(\alpha)=\alpha$. Note also that in this case we have $d-3-\alpha=z$, hence any curve with minimal node postulation (4.3) must have $e=z$.

From (16) one computes immediately:

$$
\delta(\Delta)=\frac{(v+2 z+1)(v+2 z+2)}{2}+(z+1)^{2} .
$$


Proposition 5.5. (a) Let $S \subseteq \mathbb{P}^{3}$ be a smooth cubic surface. Then for every pair of integers $(v, z)$ with $v \geq 0$ and $z \geq-1$ there is a smooth connected curve $C \subseteq S$ having minimal node postulation (16).

(b) Conversely any smooth curve $C \subseteq \mathbb{P}^{3}$ having minimal node postulation (16) with $z \geq 2$ and $d>12$ lies on a cubic surface.

Proof. We use [13], Ch. V, $\S 4$ as a basic reference for the geometry of the cubic surface. We begin by showing that $S$ contains a smooth rational curve $C^{\prime}$ of degree $v+1$. Indeed (notation as in 13]) it is sufficient to consider a general element of the linear system

$$
\Sigma:= \begin{cases}\left|\frac{v}{2} l-\left(\frac{v}{2}-1\right) e_{1}\right| & \text { if } v \text { is even } \\ \left|\frac{v+1}{2} l-\left(\frac{v-1}{2}\right) e_{1}-e_{2}\right| & \text { if } v \text { is odd }\end{cases}
$$

This shows our claim for $z=-1$. Assume now $z \geq 0$ and let $H$ be a hyperplane divisor on $S$. Then the linear system $\left|C^{\prime}+(z+1) H\right|$ is very ample by [13], V.4.12, whence a general curve $C \in\left|C^{\prime}+(z+1) H\right|$ is smooth and irreducible.

We claim that $C$ has the required properties. Indeed one computes easily that $C$ has degree $v+3 z+4=d$ and genus $g=\frac{z(z+1)}{2}+(z+1)(v+3)$, whence $\delta=\delta(\Delta)$ by (17). Then by Theorem $4.2 C$ has minimal node postulation provided it has the right speciality, namely $e(C)=z$.

Now since $-H$ is a canonical divisor for $S$ we have that $\left(C^{\prime}+z H\right) \cdot C$ is a canonical divisor of $C$, whence $e(C) \geq z$. Then we need to show that $\left(C^{\prime}-H\right) \cdot C$ is non-effective.

From the exact sequence

$$
0 \rightarrow \mathcal{O}_{S}(-H) \rightarrow \mathcal{O}_{S}\left(C^{\prime}-H\right) \rightarrow \mathcal{O}_{C^{\prime}}\left(\left(C^{\prime}-H\right) \cdot C^{\prime}\right) \rightarrow 0,
$$

recalling that $C^{\prime}$ is rational and that $\left(C^{\prime}-H\right) \cdot C^{\prime}$ is a canonical divisor of $C^{\prime}$, we get $H^{0}\left(\mathcal{O}_{S}\left(C^{\prime}-H\right)\right)=0$. Hence from the exact sequence

$$
0 \rightarrow \mathcal{O}_{S}\left(-C+C^{\prime}-H\right) \rightarrow \mathcal{O}_{S}\left(C^{\prime}-H\right) \rightarrow \mathcal{O}_{C}\left(\left(C^{\prime}-H\right) \cdot C\right) \rightarrow 0,
$$

we get $H^{0}\left(\mathcal{O}_{C}\left(\left(C^{\prime}-H\right) \cdot C\right)\right)=0$, since $H^{1}\left(\mathcal{O}_{S}\left(-C+C^{\prime}-H\right)\right)=H^{1}\left(\mathcal{O}_{S}(-(z+2) H)=0\right.$. This concludes the proof of (a).

To prove (b) let $C$ be a curve such that $\partial h_{N}=\Delta$ with $z \geq 2$. Then we have $\partial h_{N}(d-5)=$ 5 and $\partial h_{N}(d-4)=3$. On the other hand, since $d>12$, we have:

$$
\left(\begin{array}{c}
4-1 \\
2
\end{array}\right)<d-(d-4)-1+\partial h_{N}(d-4)<d-\left(\begin{array}{l}
4 \\
2
\end{array}\right)
$$

whence $h^{0}\left(\mathcal{I}_{C}(3)\right)>0$ by Theorem 3.5.

Remark 5.6. The curves with minimal node postulation we produce in the proof of Proposition 5.5 are, by construction, bilinked to the rational curve $C^{\prime}$, whence they have, up to shift, the same first cohomology of $C^{\prime}$ (see e.g. [14]). In particular if $v+1=$ $\operatorname{deg}\left(C^{\prime}\right) \geq 4$ all our curves are not aCM.

It would be interesting to know if there are aCM curves of the same degrees and specialities, having minimal node postulation. 
5.3. The case $s=4$. Now we consider the case $s=4$. The case where $\alpha$ is minimal and $C$ has the minimal node function is yet covered by Theorem 4.10(ii). Hence we may assume that $\alpha$ is not minimal, namely $\alpha>\left\lceil\frac{3 d}{4}\right\rceil-3$. We shall also assume that $\alpha \leq d-5$, otherwise the meaningful part of the node function, from $\alpha$ to $d-3$, is too short and everything trivializes. Then the minimal node function can be written as follows, for suitable integers $v \geq 1$ and $z \geq 1$ :

$$
\Delta: \ldots, 0,1, \ldots, v+3 z+3,3 z+3, \ldots, 6,3,1,0, \ldots
$$

Here $d=v+4 z+7$ and $\alpha=3 z+v+3$. Note that $d-3-\alpha=z+1$, whence if a curve has minimal node postulation then necessarily $e=z+1$.

From (18) one computes immediately:

$$
\delta(\Delta)=\frac{(v+3 z+3)(v+3 z+4)}{2}+\frac{3(z+1)(z+2)}{2}+1
$$

Proposition 5.7. (a) For every pair of integers $(v, z)$ with $v \geq 1$ and $z \geq 1$ there are a smooth quartic surface $S \subseteq \mathbb{P}^{3}$ and a smooth connected curve $C \subseteq S$ having minimal node postulation (18).

(b) Conversely any smooth curve $C \subseteq \mathbb{P}^{3}$ having minimal node postulation (18) with $z \geq 2$ and $d>20$ lies on a quartic surface.

Proof. The proof is similar to the previous one. By [15] there are a smooth quartic surface $S$ and a smooth connected curve $C^{\prime} \subseteq S$ with the following properties:

(i) $C^{\prime}$ has genus $v$ and degree $v+3$;

(ii) $\operatorname{Pic}(S)$ is freely generated by the classes of $C^{\prime}$ and $H$, where $H$ is a hyperplane divisor.

Since $K_{S} \sim 0$ we have $D^{2}=2 p_{a}(D)-2$ for every divisor $D$. In particular $C^{\prime 2}=$ $2 v-2 \geq 0$, so that $\left|C^{\prime}\right|$ has no base points, whence $\left|C^{\prime}+(z+1) H\right|$ is very ample. Then a general $C \in\left|C^{\prime}+(z+1) H\right|$ is smooth and connected and we will show that $C$ has the required properties.

First of all one computes $\operatorname{deg}(C)=v+4 z+7$ and $g=v+(z+1)(v+3)+2(z+1)^{2}$, whence $\delta=\delta(\Delta)$ by (19).

It remains to show that $C$ has the required speciality, namely $e(C)=z+1$. It is clear that $e(C) \geq z+1$ and then we have to show that the linear system $\left|C^{\prime}-H\right|$ is non-effective (see proof of Proposition 5.5).

We assume then $\left|C^{\prime}-H\right|$ effective and we seek for a contradiction.

Since $\left(C^{\prime}-H\right)^{2}=-4$ this linear system must have a fixed integral curve $E$ with negative self-intersection. It follows that $E$ is rational and $E^{2}=-2$, whence, in particular, $|E| \neq\left|C^{\prime}-H\right|$. Then if $p:=\operatorname{deg}(E)$ we have $p \leq v-2$. On the other hand, by (ii) above, there are uniquely determined integers $x$ and $y$ such that $E \sim x C^{\prime}+y H$, whence:

$$
(v+3) x+4 y=p
$$

and

$$
2(v-1) x^{2}+2(v+3) x y+4 y^{2}=-2
$$


Squaring (20) and subtracting (21) multiplied by 4 we get

$$
x^{2}=\frac{p^{2}+8}{(v+3)^{2}-8(v-1)},
$$

whence $x^{2}<1$ by a simple computation, remembering that $p \leq v-2$. This is a contradiction and the proof of (a) is complete.

Now we prove (b). Let $\Delta$ be as in (18) with $z \geq 2$ and let $C$ be a curve such that $\partial h_{N}=\Delta$. Then we have $\partial h_{N}(d-6)=9$ and $\partial h_{N}(d-5)=6$. On the other hand, since $d>20$, we have:

$$
\left(\begin{array}{c}
5-1 \\
2
\end{array}\right)<d-(d-5)-1+\partial h_{N}(d-5)<d-\left(\begin{array}{l}
5 \\
2
\end{array}\right)
$$

whence $h^{0}\left(\mathcal{I}_{C}(4)\right)>0$ by Theorem 3.5 ,

The next example shows that there are smooth quartic surfaces, other than the Mori surfaces, containing curves with minimal nodal postulation.

Example 5.8. Let $C$ be directly linked to a pair $Y$ of skew lines by surfaces of degree 4,5 . One computes $d=18, s=4$ (via the mapping cone) and one gets $\alpha$ reminding that $\omega_{C}$ is cut by surfaces of degree $5+4-4=5$ passing through $Y$ (outside $Y$ ) whence $e=3$, because $Y$ lies on a quadric. It follows that $\alpha=12$. Moreover $h^{1}\left(\mathcal{I}_{C}(1)\right)=h^{1}\left(\mathcal{I}_{Y}(4)\right)=0$ by liaison, whence $h^{0}\left(\mathcal{O}_{C}(1)\right)=4$ and $n=3$

The minimal node function $\Delta$ is:

$$
\ldots, 0,1,2,3,4,5,6,7,8,9,10,11,12,9,6,3,1,0, \ldots
$$

and its sum is $\delta(\Delta)=97$. On the other hand by liaison we have $g=39$ whence $\delta=97$. Then $C$ has minimal nodal postulation by Corollary 4.4 .

Observe also that in this case $\alpha$ is not minimal (the least value being 11), in agreement with Theorem 4.10 .

Note also that the quartic surface cannot be one of the surfaces considered in the proof of Proposition [5.7. because the classes of the two skew lines and of a hyperplane divisor are easily seen to be linearly independent.

Remark 5.9. If $v>3$ the curves we produce in the proof of Proposition [5.7 are not aCM. Indeed the starting curve $C^{\prime}$ of degree $v+3$ and genus $v$ has non trivial $H^{1}\left(\mathcal{I}_{C^{\prime}}(2)\right)$ for $v>3$, and then we can use a bilinkage argument as in Remark 5.6.

\section{REFERENCES}

[1] R. Accola, On Castelnuovo's inequalities for algebraic curves. Trans. Amer. Math. Soc. 251 (1979), 357-373.

[2] E. Arbarello, E. Sernesi, The equation of a plane curve. Duke Math. J. 46 (1979), 469-485.

[3] C. Ciliberto, Alcuni aspetti della classificazione delle famiglie di curve in uno spazio proiettivo, Boll. UMI (7) 10-A (1996), 491-535

[4] L. Chiantini, C. Ciliberto, Towards a Halphen theory of linear series on curves. Trans. Amer. Math. Soc. 351 (1999), 2197-2212. 
[5] L. Chiantini, C. Ciliberto, V. di Gennaro, The genus of projective curves. Duke Math. J. 70 (1993), 229-245.

[6] L. Chiantini, C. Ciliberto, V. di Gennaro, The genus of curves in $\mathbb{P}^{4}$ satisfying certain flag condition. Manuscr. Math. 88 (1995), 119-134.

[7] N. Chiarli, S. Greco, R. Notari, Postulation of adjoint ideals and geometry of projective curves. "Algebra, Arithmetic and Geometry with Applications: Papers from Shreeram S. Abhyankar's 70th Birthday Conference", Springer-Verlag (to appear)

[8] C. Ciliberto, R. Lazarsfeld, On the uniqueness of certain linear series on the curves of some class. Springer Lect. Notes 1092 (1984), 198-213.

[9] M. Coppens, R. Kato, Non trivial linear systems on singular plane curves. Math. Nachr. 166 (1994), 71-82.

[10] E. D. Davis, A. V. Geramita, F. Orecchia, Gorenstein algebras and the Cayley-Bacharach Theorem. Proc. Amer. Math. Soc. 93 (1985), 593-597

[11] L. Gruson, C. Peskine, Genre des courbes dans l'espace projectif. Springer Lect. Notes 687 (1978), 31-59.

[12] J. Harris, D. Eisenbud, Curves in projective spaces. Montreal University Press (1982).

[13] R. Hartshorne, Algebraic Geometry, Springer-Verlag (1977).

[14] J. C. Migliore, Introduction to liaison theory and deficiency modules, Progress in Math. 165, Birkhäuser, 1998

[15] S. Mori, On degrees and genera of curves on smooth quartic surfaces in $\mathbb{P}^{3}$. Nagoya Math. J. 96 (1984), 127-132

[16] E. Schlesinger, A speciality theorem for Cohen-Macaulay space curves, Trans. Amer. Math. Soc. 351 (1999), 2731-2743

${ }^{1}$ Dipartimento di Matematica, Università di Siena, I-53100 Siena, Italy

E-mail address: CHIANTINI@UNISI.IT

2 Dipartimento di Matematica, Politecnico di Torino, I-10129 Torino, Italy

E-mail address: NADIA.CHIARLI@POLITO.IT

3 Dipartimento di Matematica, Politecnico di Torino, I-10129 Torino, Italy

E-mail address: SILVIO.GRECO@POLITO.IT 